\title{
Adrenalectomy does not improve survival rates of patients with solitary adrenal metastasis from non-small cell lung cancer
}

This article was published in the following Dove Press journal:

Therapeutics and Clinical Risk Management

23 March 2017

Number of times this article has been viewed

\author{
Shao-Hong Huang ${ }^{1, *}$ \\ Qing-Lei Kong',* \\ Xue-Xia Chen ${ }^{3}$ \\ Jin-Yuan $\mathrm{He}^{1}$ \\ Jie Qin ${ }^{4}$ \\ Zhuang-Gui Chen ${ }^{5,6}$
}

'Department of Cardiothoracic Surgery, The Third Affiliated Hospital, Sun Yat-sen University, Guangzhou, China; ${ }^{2}$ Department of Emergency, the Third Affiliated Hospital, Sun Yat-sen University, Guangzhou, China; ${ }^{3}$ Department of Nursing, Eastern Hospital of The First Affiliated Hospital, Sun Yat-sen University, Guangzhou, China; ${ }^{4}$ Department of Radiology, The Third Affiliated Hospital, Sun Yat-sen University, Guangzhou, China; ${ }^{5}$ Department of Pediatrics, Pediatric Intensive Care Unit, The Third Affiliated Hospital of Sun Yat-sen University, ${ }^{6}$ nstitution of Respiratory Disease of Sun Yat-sen University, Guangzhou, China

*These authors contributed equally to this work
Background and purpose: Several case reports and studies have suggested that there is an increased survival rate for patients who undergo resection of solitary adrenal metastasis from non-small cell lung cancer (NSCLC). This study aimed to investigate whether NSCLC patients with solitary adrenal metastasis could gain a higher survival rate after adrenalectomy (ADX) when compared with those patients undergoing nonsurgical treatment, and to investigate the potential prognostic factors.

Patients and methods: A total of 1,302 NSCLC inpatients' data from 2001 to 2015 were retrospectively reviewed to identify those with solitary adrenal metastasis. Overall survival for those who underwent both primary resection and ADX was compared to those patients with conservative treatment using the log-rank test. Potential prognostic variables were evaluated with univariate and multivariate analyses including clinical, therapeutic, pathologic, primary and metastatic data.

Results: A total of 22 NSCLC patients with solitary adrenal metastasis were identified, with an overall median survival of 11 months (95\% confidence interval: 9.4-12.6 months) and a 1-year survival rate of $51.4 \%$ (95\% confidence interval: $29.6 \%-73.2 \%$ ). All of the patients had died by 30 months. There was no significant survival difference between patients who underwent primary and metastasis resection $(\mathrm{n}=10)$ and those treated conservatively $(\mathrm{n}=12),(P=0.209)$. Univariate analysis identified Eastern Cooperative Oncology Group performance status (ECOG PS) as the significant predictor of survival ( $P=0.024)$. Age ( $<65 \mathrm{vs} \geq 65$ years), sex, pathologic type, mediastinal lymph node stage ( $\mathrm{N} 2 \mathrm{vs} \mathrm{N} 0 / \mathrm{N} 1)$, primary tumor size $(<5 \mathrm{vs} \geq 5 \mathrm{~cm})$, primary location (central vs peripheral), metastatic tumor size $(<5 \mathrm{vs} \geq 5 \mathrm{~cm})$, metastasis laterality, synchronous metastasis, and metastatic field radiotherapy were not identified as potential prognostic factors in relation to survival rate. In multivariate analysis, a stepwise selection procedure allowed both ECOG PS $(P=0.007$, relative risk $=3.57)$ and pathologic type $(P=0.069)$ to enter the Cox's hazard function.

Conclusion: Primary and metastatic radical resection may not prolong the survival of NSCLC patients with solitary adrenal metastasis. ECOG PS and pathologic type might be the prognostic factors for these patients.

Keywords: adrenalectomy, solitary metastasis, non-small cell lung cancer, survival, prognostic factors

\section{Introduction}

Distant metastasis from non-small cell lung cancer (NSCLC) denotes a poor prognosis in most patients who are not amenable to curative treatment, with a median survival rate of less than 11 months. ${ }^{1}$ Isolated extra pulmonary metastasis may not always be 
like this, however, an aggressive surgical approach might result in an improved overall survival. ${ }^{2}$ Several reports have suggested an increased survival for selected NSCLC patients who underwent resection of solitary adrenal metastasis, with a 5-year survival rate exceeding 25\%., Because of the rarity of solitary adrenal metastasis, ${ }^{5,6}$ high variability among clinical studies and selection bias, ${ }^{2}$ the indications and effectiveness for adrenalectomy (ADX) in the subset of NSCLC patients have not been clearly defined. The aims of this study were to review our experience with treatment of solitary adrenal metastasis from NSCLC during the past 10 years, to investigate the outcomes of patients who underwent ADX compared with those treated nonoperatively, and to analyze the clinical and oncological factors that may influence the survival rate.

\section{Patients and methods Patients and data collection}

A prospectively maintained electric inpatient database was reviewed to identify patients with solitary adrenal metastasis from NSCLC between January 2001 and December 2015. When necessary, data from the database were verified and supplemented by a retrospective chart review and most recent physician interviews by telephone. The inclusion criteria were: 1) definitive pathologic diagnosis of pulmonary lesion via bronchoscopy, core needle biopsy (under radiological guidance or thoracoscopy), or surgical specimens; 2) single adrenal metastatic lesion was detected; and 3) no other suspicious sites for metastatic disease on imaging (including chest X-ray, abdominal ultrasonography, chest and abdominal computed tomography, bone scan, positron emission tomography and brain magnetic resonance imaging for the patients had neurologic symptoms). Patient medical records were reviewed for demographics, timing of diagnosis of primary disease and metastasis, type of primary tumor, characteristics of primary and metastatic lesion, therapeutic interventions, survival status, and length of follow-up. The duration of follow-up was calculated from the time of ADX or date of the pathologic diagnosis of lung cancer in patients treated without ADX to the time of recurrence, death, or date of last follow-up. Pathology of the primary lesion was acquired via both cytology (bronchoscopic or core needle biopsy) and surgical specimens.

During this period, 1,302 NSCLC patients were treated in our department and $22(1.7 \%)$ patients with solitary adrenal metastasis (no lesions at other sites detected on imaging) were identified. The patients included 16 males and 6 females with a median age of 59 years (range: 32-84 years).
Ten of the 22 patients received ADX (open or laparoscopic). In all patients who underwent ADX, the primary NSCLC had been treated by complete resection (open or thoracoscopic). Besides the imaging checkups listed above, preoperative workup also included electrocardiogram, cardiac Doppler, spirometry, bronchoscopy, and lung perfusion scan, which were not arbitrary for the conservatively treated patients. Among patients who underwent adrenalectomy, $5(50 \%)$ received neoadjuvant chemotherapy before primary resection. All 10 patients received adjuvant chemotherapy and 2 of them received concurrent radiation therapy to the metastatic lesions after curative ADX. All 12 patients treated nonoperatively received 4-6 cycles of platinumbased systemic chemotherapy and 4 (33\%) of them received palliative radiotherapy to the metastatic lesion. None of the patients underwent thoracic radiotherapy.

We also concluded the reasons why adrenal resection was not part of the treatment for the group of patients managed nonoperatively, which included: mediastinal nodal N3 and melting N2 disease $(n=3)$, poor lung function (forced expiratory volume in one second $<40 \%)(n=4)$, severe comorbid illness $(n=2)$, and patient preference $(n=1)$. For some patients, it was unclear why they did not receive surgical resection $(n=2)$.

Performance status was recorded as the Eastern Cooperative Oncology Group performance status (ECOG PS) determined by reviewing the electric inpatient database for the patient's first time admission. Adrenal metastases were regarded as synchronous ( $<6$ months), or metachronous ( $\geq 6$ months), depending on the interval after the initial diagnosis of the primary lung cancer. Overall survival (OS) was defined as the interval from the date of ADX or the initial diagnosis of NSCLC in conservatively treated patients, to the date of last follow-up or death.

This study gained the approval from the Ethics Committees of Guangdong General Hospital and The Third Affiliated Hospital of Sun Yat-sen University. All the patients enrolled in our study were informed and have given their consents for reviewing their medical records.

\section{Statistical analysis}

Categorical variables were compared by the Fisher's exact test. Continuous variables were compared by the Student's $t$-test. Survival probabilities were estimated using the Kaplan-Meier method. The significance of differences between subgroups was calculated using the log-rank test. Associations of clinical, surgical, and pathologic features with OS were evaluated using Cox proportional regression models (forward likelihood ratio). Each variable in the 
model was sequentially entered to assess for confounding by noting changes in the hazard coefficient and $P$-value to include or exclude a variable. All statistical analyses were performed using SPSS release 19.0 software (SPSS Inc, Chicago, IL, USA). All tests were two-sided, and $P$-values of less than 0.05 were considered statistically significant.

\section{Results}

\section{Clinical characteristics and baseline analysis}

The median follow-up period for the entire group (censored patients) was 10 months (range: 5-29 months). At the end of the study, 2 patients were alive and 2 patients were lost to follow-up. The median interval time between surgical resection of the primary lung cancer and resection of the adrenal metastasis was one month (range: $0.5-4$ months). No patient experienced any serious post-operative complication and there was no operation-related death. Relevant clinical and pathologic characteristics are summarized in Table 1.

Table I Clinical and pathologic features of study cohort

\begin{tabular}{|c|c|c|c|}
\hline Characteristics/variables & $\begin{array}{l}\text { ADX } \\
(n=10)\end{array}$ & $\begin{array}{l}\text { Nonsurgical } \\
(n=12)\end{array}$ & $P$-value \\
\hline Age (years), mean $\pm S D$ & $56 \pm 14$ & $62 \pm 12$ & 0.273 \\
\hline Sex, n (\%) & & & 0.583 \\
\hline Male & $7(70)$ & $9(75)$ & \\
\hline ECOG PS, n (\%) & & & 0.108 \\
\hline 0 & $3(30)$ & $2(16.7)$ & \\
\hline 1 & $6(60)$ & $3(25)$ & \\
\hline 2 & $I(10)$ & $6(50)$ & \\
\hline 3 & 0 & I (8.3) & \\
\hline Histology, n (\%) & & & 0.86 \\
\hline Squamous cell carcinoma & $2(20)$ & I (8.3) & \\
\hline Adenocarcinoma & $6(60)$ & $6(6 I)$ & \\
\hline Adenosquamous carcinoma & $I(10)$ & I (8.3) & \\
\hline Large cell carcinoma & 0 & I (8.3) & \\
\hline Unclassified carcinoma & $\mathrm{I}(\mathrm{I0})$ & $3(25)$ & \\
\hline Mediastinal $\mathrm{N}$ stage, $\mathrm{n}(\%)$ & & & 0.078 \\
\hline No & $I(10)$ & 0 & \\
\hline NI & $2(20)$ & 0 & \\
\hline N2 & $7(70)$ & $12(100)$ & \\
\hline Primary location, n (\%) & & & 0.667 \\
\hline Central & $5(50)$ & $4(33.3)$ & \\
\hline $\begin{array}{l}\text { Primary diameter }(\mathrm{cm}), \\
\text { mean } \pm S D\end{array}$ & $3.5 \pm 2.4$ & $4.2 \pm 1.7$ & 0.462 \\
\hline Metastasis laterality, n (\%) & & & 0.204 \\
\hline Ipsilateral & $8(80)$ & $6(50)$ & \\
\hline Metastasis interval, n (\%) & & & 0.652 \\
\hline Synchronous ( $<6$ months) & $6(60)$ & $9(75)$ & \\
\hline $\begin{array}{l}\text { Metastatic diameter }(\mathrm{cm}) \\
\text { mean } \pm \text { SD }\end{array}$ & $3.3 \pm 1.6$ & $4.1 \pm 1.4$ & 0.247 \\
\hline $\begin{array}{l}\text { Median survival } \\
\text { (months), range }\end{array}$ & $12(9.1-14.9)$ & $9(6.9-11.1)$ & 0.209 \\
\hline
\end{tabular}

Abbreviations: ADX, adrenalectomy; ECOG PS, Eastern Cooperative Oncology Group performance status; SD, standard deviation.
Sex, age, performance status, lymph node involvement, histology, size, and location of the primary and metastatic tumor were similar between groups. In this study, 19 (86\%) patients had N2 disease for the whole cases and the proportion of patients who underwent ADX tended to be lower than patients treated conservatively, but this was not statistically significant $(P=0.078)$.

\section{Univariate survival analysis}

Estimated median survival was 11 months (95\% confidence interval [CI]: 9.4-12.6 months) and 1-year survival rate was 51.4\% (95\% CI: $29.6 \%-73.2 \%$ ) for all 22 patients (Figure 1). On the univariate analysis, ECOG PS was the only significant predictor of survival $(P=0.024)$. The median survival and $95 \%$ CI for patients with ECOG PS 0, 1, 2, and 3 were 14 months (7.6-20.4 months), 11 months (5.9-16.1 months), 8 months (5.6-10.4 months), 6 months (not available), respectively. Cross comparisons showed a significant difference between patients with an ECOG PS of 0 and $2(P=0.047)$ and $3(P=0.025)$, and 2 and $3(P=0.008)$ (Figure 2$)$. The median survival tended to be longer in patients undergoing ADX compared with those treated nonsurgically (12 months vs 9 months, $P=0.209)$. Histology was not the significant prognostic factor $(P=0.052)$, but cross comparisons showed a significant difference between adenocarcinoma and adenosquamous carcinoma $(P=0.006)$ (Figure 3$)$. All other factors analyzed, including age, sex, nodal involvement, radiotherapy, primary and metastatic characteristics, did not influence survival (Table 2). Neoadjuvant chemotherapy was not a significant predictor of survival among patients treated surgically $(P=0.430)$.

\section{Multivariate survival analysis}

In the multivariable analysis, a stepwise selection procedure identified ECOG PS $(P=0.007$, relative risk $=3.57)$ and tumor histology $(P=0.069)$ as independent factors of prognostic value. Age, sex, nodal involvement, radiotherapy, primary location, metastasis laterality, primary and metastatic diameter were not associated with prognosis (Table 2). For patients treated surgically, interval time between primary resection and ADX was not the prognostic factor $(P=0.366)$, either.

\section{Discussion}

The adrenal gland is one of the most common organs involved in metastatic disease, with postmortem cases reporting the incidence as high as $38 \% .^{7}$ However, singular adrenal metastases in patients with NSCLC are extremely rare, representing less than $2 \%$ of surgical cases. ${ }^{5,6}$ Solitary adrenal 
A

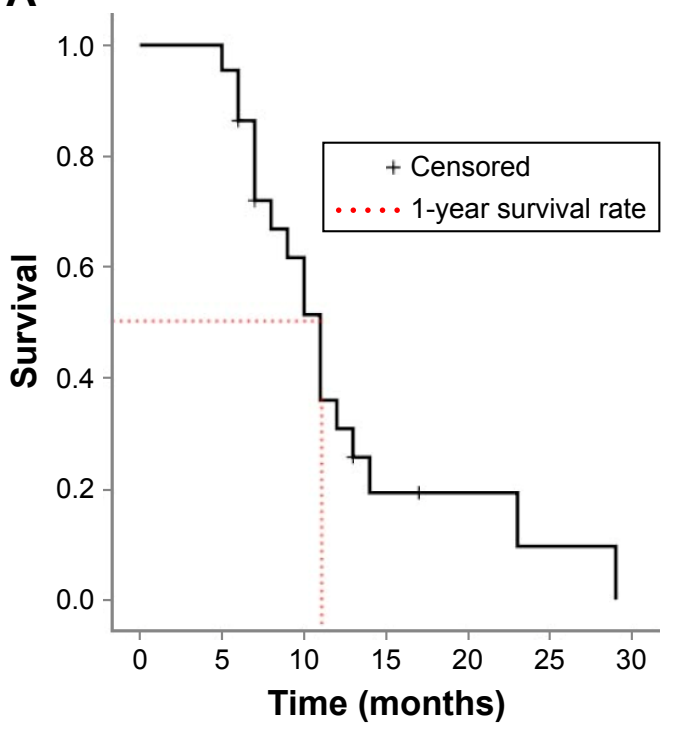

B

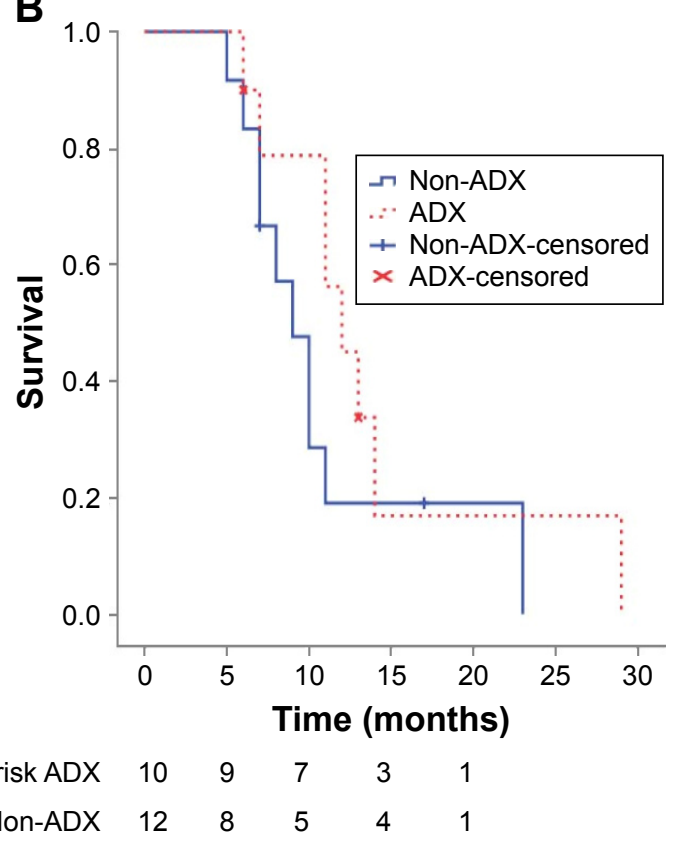

Figure I Kaplan-Meier survivor curves for all patients enrolled $(\mathbf{A})$ and for both groups (B).

Abbreviations: ADX, adrenalectomy; Non-ADX, nonsurgical treatment.

metastasis may be observed in many primaries, such as NSCLC. Although there is only $17 \%$ of isolated adrenal metastasis in NSCLC patients, they are considered to have the worst prognosis, ${ }^{8}$ showing a median survival between 6 and 9 months when treated nonsurgically. ${ }^{6,9}$ Metastatic lesions are indications of a disseminated disease, which generally

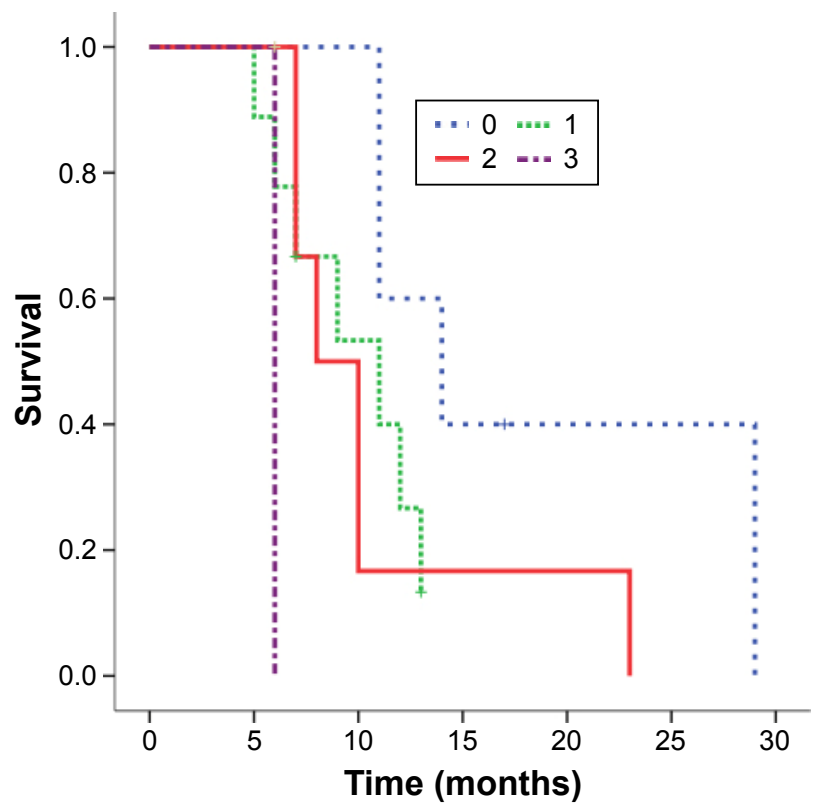

Figure 2 Kaplan-Meier survivor curve comparing overall survival among patients with different ECOG PS.

Abbreviation: ECOG PS, Eastern Cooperative Oncology Group performance status. excludes the aggressive treatment options. However, the success of aggressive resection for solitary cerebral metastasis makes surgeons reconsider this issue. Case reports and studies have suggested an increased survival for patients who undergo resection of solitary adrenal metastasis from NSCLC. ${ }^{5,10-13}$ Vazquez et al reported their 21 patients who underwent resection of solitary adrenal metastasis from NSCLC had a median survival of 3.5 years and 1-year survival of $91 \%{ }^{7}$ Mercier et al showed a median survival of 13 months and a 5 -year survival of $23 \%$ for 23 patients. ${ }^{4}$

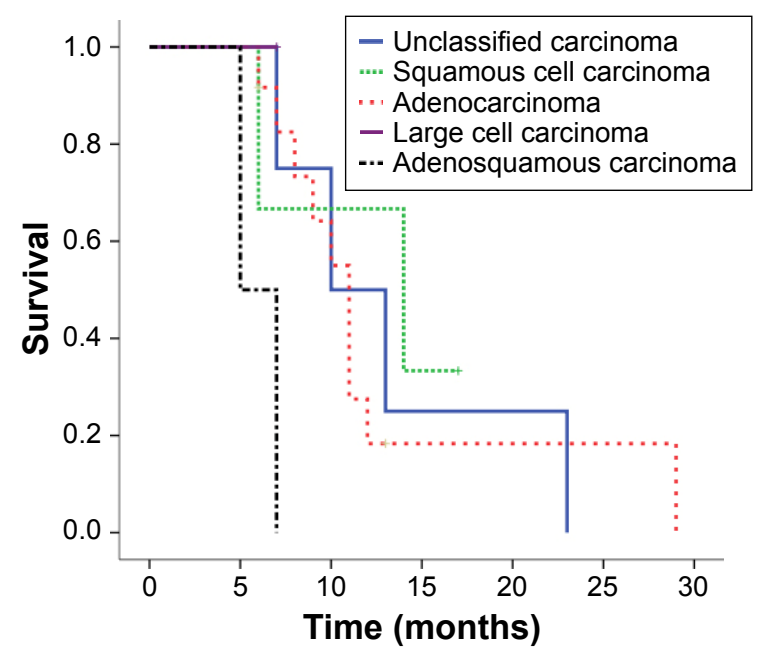

Figure 3 Kaplan-Meier survivor curve comparing overall survival among patients with different histology. 
Table 2 Variables not entered into proportional regression function and $P$-value

\begin{tabular}{lll}
\hline Variables & $\begin{array}{l}\text { Univariate } \\
\text { analysis }\end{array}$ & $\begin{array}{l}\text { Multivariate } \\
\text { analysis }\end{array}$ \\
\hline Age & 0.27 I ( $\geq 65$ vs $<65$ years $)$ & 0.355 \\
Sex & 0.138 & 0.558 \\
N stage & 0.825 & 0.932 \\
Primary diameter & $0.091(\geq 5$ vs $<5 \mathrm{~cm})$ & 0.131 \\
Primary location & 0.766 & 0.705 \\
Metastatic diameter & $0.562(\geq 5$ vs $<5 \mathrm{~cm})$ & 0.256 \\
Metastasis laterality & 0.490 & 0.278 \\
Metastasis interval & 0.353 & 0.534 \\
ADX & 0.209 & 0.448 \\
Radiotherapy & 0.964 & 0.880 \\
\hline
\end{tabular}

Abbreviation: ADX, adrenalectomy.

In the meta-analysis by Tanventayon et al which compiled reports from 10 articles with 114 NSCLC cases who underwent ADX, the median survival exceeded 12 months and 5-year survival was $25 \% .{ }^{3}$ Porte et al collected 43 cases from several centers in France, with 17 of them presenting with N2 disease, showed a median survival of 11 months and 3 cases survived over 5 years. ${ }^{5}$

In our study, the median survival ( 9 months) for patients treated nonsurgically was comparable with previous reports. ${ }^{6,9}$ Whereas, for those who underwent ADX, the median survival was only 12 months and no long-term survival occurred, which seems a very poor prognosis compared with other reports. ${ }^{3,4,7}$ Mediastinal nodal status is recognized as a powerful impact factor on survival for patients with NSCLC. A relatively high proportion of $\mathrm{N} 2$ disease in the operative group (70\%) may account for the poor result for the patients after ADX, compared with $17 \%-40 \%$ in other published studies. ${ }^{2,4,5}$

Several retrospective studies have indicated that adrenalectomy may bring survival advantage to the patients with solitary adrenal metastases, however, most of them were non-controlled studies. .,5, $, 10,11$ In several controlled studies, the results were not consistent. ${ }^{2,9}$ In our study, it seems that surgical resection had a survival advantage compared with nonoperative management (median survival, 12 vs 9 months), although this was an insignificant statistical difference $(P=0.209)$. Similar to Mordant et al's study, ${ }^{2}$ our study data did not demonstrate the improved survival in the aggressive surgical group compared with conservative regime. This result needs to be interpreted with caution due to the high proportion (86\%) of $\mathrm{N} 2$ disease in all patients enrolled, which might blur the comparison in survival outcome. Therefore, it is hard to determine the effect of ADX on survival of NSCLC patients when considering the confounding factors such as nodal status, and small sample in this study.
For a long time, ECOG PS has been one of the most important survival predictor and key point for the regiment decision for advanced NSCLC. Surprisingly, there was no study focusing on its predictive role in ADX for the special patient subset. Our data showed that poor ECOG PS was still a powerful negative predictor of prognosis for patients with solitary adrenal metastases, with a 3.57-fold risk increase (relative risk) per score increment. Therefore, we propose aggressive resections should not be encouraged for patients with a poor performance status. Histology was another possible survival predictor in our series, with a marginal significance in both univariate $(P=0.052)$ and multivariate analysis $(P=0.069)$. Mordant et al considered adenocarcinoma as a predictor of favorable prognosis for the solitary metastasis, ${ }^{2}$ but other reports did not demonstrate the survival difference between histologic subtypes. ${ }^{5,11,12,14}$

\section{Conclusion}

Because of the rarity of solitary adrenal metastases from NSCLC, like all previously published studies, our retrospective study was relatively small, inducing a weak power. Beside selection bias, including a high proportion of N2 involvement, this study failed to demonstrate the advantage of surgical resection over nonsurgical treatments. With respect to these limitations, this study indicated ECOG PS as an important prognostic factor for patients with solitary adrenal metastases from NSCLC. Our experience suggests that aggressive surgical resections of primary and adrenal lesion may not be appropriate for patients with a poor performance status and $\mathrm{N} 2$ disease.

\section{Acknowledgment}

This study was supported by the National Natural Science Foundation of China (grant no 81470219) and Science and Technology Projects Foundation of Guangdong Province (grant nos 2016A020215077, 2014A020212482, and 2014A020212120).

\section{Author contributions}

Shao-Hong Huang and Qing-Lei Kong designed the study and participated in the drafting of the manuscript. Xue-Xia Chen and Jin-Yuan He participated in the data collection and revisions of the draft. Jie Qin participated in the data collection and manuscript interpretation. Zhuang-Gui Chen designed the study, drafted the manuscript and participated in the critical revisions of the draft. All authors contributed toward data analysis, drafting and critically revising the paper and agree to be accountable for all aspects of the work. 


\section{Disclosure}

The authors report no conflicts of interest in this work.

\section{References}

1. Woodard GA, Jones KD, Jablons DM. Lung cancer staging and prognosis. Cancer Treat Res. 2016;170:47-75.

2. Mordant P, Arame A, De Dominicis F, et al. Which metastasis management allows long-term survival of synchronous solitary M1b non-small cell lung cancer? Eur J Cardiothorac Surg. 2012;41(3):617-622.

3. Tanvetyanon T, Robinson LA, Schell MJ, et al. Outcomes of adrenalectomy for isolated synchronous versus metachronous adrenal metastases in non-small-cell lung cancer: a systematic review and pooled analysis. J Clin Oncol. 2008;26(7):1142-1147.

4. Mercier O, Fadel E, de Perrot M, et al. Surgical treatment of solitary adrenal metastasis from non-small cell lung cancer. J Thorac Cardiovasc Surg. 2005;130(1):136-140.

5. Porte H, Siat J, Guibert B, et al. Resection of adrenal metastases from nonsmall cell lung cancer: a multicenter study. Ann Thorac Surg. 2001;71(3): 981-985.

6. Griffioen GH, Toguri D, Dahele M, et al. Radical treatment of synchronous oligometastatic non-small cell lung carcinoma (NSCLC): patient outcomes and prognostic factors. Lung Cancer. 2013;82(1):95-102.

7. Vazquez BJ, Richards ML, Lohse CM, et al. Adrenalectomy improves outcomes of selected patients with metastatic carcinoma. World J Surg. 2012;36(6):1400-1405.
8. Muth A, Persson F, Jansson S, Johanson V, Ahlman H, Wängberg B. Prognostic factors for survival after surgery for adrenal metastasis. Eur J Surg Oncol. 2010;36(7):699-704.

9. Raz DJ, Lanuti M, Gaissert HC, Wright CD, Mathisen DJ, Wain JC. Outcomes of patients with isolated adrenal metastasis from non-small cell lung carcinoma. Ann Thorac Surg. 2011;92(5):1788-1792; discussion 1793 .

10. Strong VE, D'Angelica M, Tang L, et al. Laparoscopic adrenalectomy for isolated adrenal metastasis. Ann Surg Oncol. 2007;14(12): 3392-3400.

11. Sebag F, Calzolari F, Harding J, Sierra M, Palazzo FF, Henry JF. Isolated adrenal metastasis: the role of laparoscopic surgery. World J Surg. 2006; 30(5):888-892.

12. Pfannschmidt J, Schlolaut B, Muley T, Hoffmann H, Dienemann H. Adrenalectomy for solitary adrenal metastases from non-small cell lung cancer. Lung Cancer. 2005;49(2):203-207.

13. Taira N, Kawabata $T$, Ichi $T$, et al. Long-term survival after surgical treatment of metachronous bilateral adrenal metastases of non-small cell lung carcinoma. Am J Case Rep. 2014;15:444-446.

14. Oshiro Y, Takeda Y, Hirano S, Ito H, Aruga T. Role of radiotherapy for local control of asymptomatic adrenal metastasis from lung cancer. Am J Clin Oncol. 2011;34(3):249-253.
Therapeutics and Clinical Risk Management

\section{Publish your work in this journal}

Therapeutics and Clinical Risk Management is an international, peerreviewed journal of clinical therapeutics and risk management, focusing on concise rapid reporting of clinical studies in all therapeutic areas, outcomes, safety, and programs for the effective, safe, and sustained use of medicines. This journal is indexed on PubMed Central, CAS,
Dovepress

EMBase, Scopus and the Elsevier Bibliographic databases. The manuscript management system is completely online and includes a very quick and fair peer-review system, which is all easy to use. Visit $\mathrm{http}: / /$ www.dovepress.com/testimonials.php to read real quotes from published authors. 